\title{
Size and PEG Length-Controlled PEGylated Monocrystalline Superparamagnetic Iron Oxide Nanocomposite for MRI Contrast Agent
}

This article was published in the following Dove Press journal: International Journal of Nanomedicine

\author{
Li-Hua Deng $\mathbb{( D}^{1,2}$ \\ Hai Jiang' \\ Fu-Lin Lu' \\ Han-Wei Wang' \\ $\mathrm{Yu} \mathrm{Pu}^{\prime}$ \\ Chang-Qiang Wu (ID) \\ Hong-Jie Tang ${ }^{3}$ \\ $\mathrm{Ye} \mathrm{Xu}^{4}$ \\ Tian-Wu Chen' \\ Jiang Zhu' \\ Cheng-Yi Shen' \\ Xiao-Ming Zhang'
}

'Medical Imaging Key Laboratory of Sichuan Province and School of Medical Imaging, Affiliated Hospital of North Sichuan Medical College, Nanchong, 637000, People's Republic of China; ${ }^{2}$ Department of Radiology, First People's Hospital of Neijiang, Neijiang 641000, People's Republic of China; ${ }^{3}$ Department of Radiology, Nanchong Hospital of Traditional Chinese Medicine, Nanchong 637000, People's Republic of China; ${ }^{4}$ Department of Radiology, Children's Hospital of Chongqing Medical University, Chongqing 40I I22, People's Republic of China

Correspondence: Chang-Qiang Wu; Xiaoming Zhang

Sichuan Key Laboratory of Medical Imaging and School of Medical Imaging, Affiliated Hospital of North Sichuan Medical College, 234th Fujiang Road, Nanchong 637000, People's Republic of China

Tel +86-13990723836

Email wucq1984@I63.com;

zhangxm@nsmc.edu.cn
Objective: PEGylated superparamagnetic iron oxide (SPIO) is the most promising alternatives to gadolinium-based contrast agents (GBCAs) in MRI. This paper is to explore the imaging effects of PEGylated SPIO, which is influenced by particle sizes and surface polyethylene glycol (PEG) coating, using as MRI contrast agents at different magnetic field intensities.

Methods: Firstly, nine PEGylated monocrystalline SPIO nanoparticles with different nanocrystal sizes and different molecular weights PEG coating were prepared, and then physical and biological properties were analyzed. Finally, MRI imaging in vivo was performed to observe the imaging performance.

Results: Nine PEGylated monocrystalline SPIO nanoparticles have good relaxivities, serum stability, and biosecurity. At the same time, they show different imaging characteristics at different magnetic field intensities. Eight-nanometer SPIO@PEG5k is an effective $T_{2}$ contrast agent at $3.0 \mathrm{~T}\left(r_{2} / r_{1}=14.0\right)$, is an ideal $T_{1}-T_{2}$ dual-mode contrast agent at $1.5 \mathrm{~T}\left(r_{2} / r_{1}=\right.$ 6.52), and is also an effective $T_{1}$ contrast agent at $0.5 \mathrm{~T}\left(r_{2} / r_{1}=2.49\right)$, while 4-nm SPIO@PEG5k is a $T_{1}-T_{2}$ dual-mode contrast agent at $3.0 \mathrm{~T}\left(r_{2} / r_{1}=5.24\right)$, and is a useful $T_{1}$ contrast agent at $0.5 \mathrm{~T}\left(r_{2} / r_{1}=1.74\right)$ and $1.5 \mathrm{~T}\left(r_{2} / r_{1}=2.85\right)$. MRI studies in vivo at 3.0 $\mathrm{T}$ further confirm that 4-nm SPIO@PEG5k displays excellent $T_{1}-T_{2}$ dual-mode contrast enhancement, whereas 8-nm SPIO@PEG5k only displays $T_{2}$ contrast enhancement.

Conclusion: PEGylated SPIOs with different nanocrystal sizes and PEG coating can be used as $T_{1}, T_{2}$, or $T_{1}-T_{2}$ dual-mode contrast agents to meet the clinical demands of MRI at specific magnetic fields.

Keywords: magnetic resonance imaging, contrast agents, superparamagnetic iron oxide, polyethylene glycol

\section{Introduction}

Gadolinium-based contrast agents (GBCAs) are widely used in clinical magnetic resonance imaging (MRI). In recent years, however, their safety has been under great concern, due to the associated nephrogenic system fibrosis (NSF) and gadolinium retention. ${ }^{1-3}$ Superparamagnetic iron oxides (SPIO) have been widely studied as MRI contrast agents for many years, because of their excellent magnetic properties and biocompatibility. ${ }^{4-7}$ SPIO-based nanoparticles demonstrate high proton relaxation efficiency and slower kidney clearance, which can be used at low concentrations compared with GBCAs. ${ }^{8}$ Meanwhile, iron, as the main component, is also one of the essential elements within the human body, and hence has 
inherent metabolic pathways in vivo. Therefore, SPIO may be considered as the most promising alternative to GBCAs in MRI.

SPIOs are small-sized iron oxide nanocrystals $\left(\mathrm{Fe}_{3} \mathrm{O}_{4}\right.$ or $\gamma-\mathrm{Fe}_{2} \mathrm{O}_{3}$ ). To obtain the colloidal stability of these inorganic nanocrystals in biological applications, surface coating with water-soluble materials is essential. ${ }^{4-7}$ Polyethylene glycol (PEG) is the most commonly used non-ionic hydrophilic polymer with good biocompatibility and has been widely applied in biopharmaceuticals as a solvent or excipient. ${ }^{9}$ PEGylation of biomolecules or drug delivery nanocarriers can increase the circulatory half-life, and reduce antigenicity as well as immunogenicity in vivo, hence improving its pharmaceutical and pharmacological properties. ${ }^{10-12}$ Over the past decade, PEGylated SPIOs have been developed by various methods, and they exhibited favorable stability in aqueous phase and biocompatibility. For example, the amphiphilic polymer based on PEG (PCL-PEG or DSPEPEG) can form micelles when in an aqueous phase and were used to encapsulate hydrophobic SPIO to form PEGylated SPIO nanoclusters. ${ }^{13-15}$ The size of these PEGylated SPIO nanoclusters ranges from tens to hundreds of nanometers and can be tuned by adjusting the ratio between the polymer and SPIO. Another method to obtain PEGylated SPIO is to modify specific ligands at the end of PEG, which chelates iron (III) onto the surface of SPIO nanocrystals. ${ }^{16-19}$

Previous research has shown that SPIO nanoclusters usually have high $T_{2}$ relaxivity $\left(r_{2}\right)$, and can only be used as $T_{2}$ contrast agents. ${ }^{8}$ However, clinical applications of $T_{2}$ contrast agents were limited due to the 'black holes' effect, which makes it difficult to distinguish between contrast enhancement lesions and low signal-to-noise ratio (SNR) areas such as calcification, air, hemorrhage, and blood clots. $^{20,21}$ Monodisperse small-sized SPIO nanocrystals suppress the $T_{2}$ effect as a result of their small magnetic moment and maintain a high $T_{1}$ relaxivity $\left(r_{1}\right)$. This kind of SPIO nanoparticles has been studied as $T_{1}$ or $T_{1}-T_{2}$ dualmode contrast agents according to their $r_{2} / r_{1}$ ratio. $^{22,23}$ Therefore, PEGylated small-sized SPIO nanocomposites have important clinical application and research value.

The relaxivities $\left(r_{2}\right.$ and $\left.r_{1}\right)$ and dynamic distributions of the SPIO nanoparticles in the body are of vital importance to its imaging effect in contrast-enhanced MRI (CEMRI) and are mainly related to the crystal size, crystal shape, surface coating, and aggregation state. ${ }^{24-26}$ It is hence of great significance to systematically study the interactive relationship between the properties and parameters of PEGylated SPIO, to facilitate advances in its development and potential applications. In this paper, we designed and prepared nine PEGylated monocrystalline SPIO nanoparticles with various nanocrystal sizes and surface PEG lengths (Figure 1A), and performed detailed studies of their relaxation properties in vitro and imaging features in vivo.

\section{Materials and Methods Synthesis of Dopamine-Modified Polyethylene Glycol (PEG-DA)}

Polyethylene glycol methyl ether (mPEG-OH) with different molecular weights $(\mathrm{Mn}=550,2 \mathrm{k}$, or $5 \mathrm{k} \mathrm{Da})$ were purchased from Sigma-Aldrich (St Louis, MO, USA). 1-mmol mPEG$\mathrm{OH}$ was placed in a $100 \mathrm{~mL}$ round bottom flask, and vacuum dried for $2 \mathrm{~h} .20 \mathrm{~mL}$ of dried tetrahydrofuran was added and stirred magnetically. Carbonyldiiazole $(810 \mathrm{mg}, 5 \mathrm{mmol})$ was added in an inert gas atmosphere, and the mixture was refluxed overnight. The resulting solution was cooled to room temperature by removing the heat source, and $100 \mu \mathrm{L}$ of water was added and stirred for $5 \mathrm{~min}$. Vacuum distillation was performed and the product obtained was dissolved in 20$\mathrm{mL}$ dichloromethane, and dropped into cold diethyl ether to obtain the precipitate (mPEG-CI).

1-mmol mPEG-CI was dissolved in $40 \mathrm{~mL}$ of tetrahydrofuran. Dopamine hydrochloride $(560 \mathrm{mg}, 3 \mathrm{mmol})$ and triethylamine $(1 \mathrm{~mL})$ were added under an inert gas atmosphere and reacted at room temperature for $24 \mathrm{~h}$. The solvent was evaporated using vacuum distillation. The product was dissolved in 20-mL dichloromethane, and dropped into cold diethyl ether to obtain the precipitate which was dried under vacuum conditions to give the white powdered product (mPEG-DA).

\section{The Preparation of PEGylated SPIO Nanoparticles (SPIO@PEG)}

SPIO nanocrystals were synthesized by thermal decomposition of iron acetylacetonate (Sigma Aldrich, St Louis, MO, USA), and the crystal size was tuned by modifying the reaction conditions (namely the reaction temperature and solvent) as previously reported. ${ }^{27}$ The detailed procedures were described in Supporting Information. The citric acid (1 g) was dissolved in $70 \mathrm{~mL}$ of ultrapure water, and $\mathrm{NaOH}$ was added to adjust the $\mathrm{pH}$ as 7.2. One hundred and ten milliliter acetone and 70-mL SPIO hexane solution (containing 50-mg nanoparticles) were added. The mixture was stirred and refluxed for $48 \mathrm{~h}$. The sodium citrate coated SPIO (SPIO@CA) was obtained by centrifugation after ligand exchange, and subsequently dispersed in ultrapure water. 


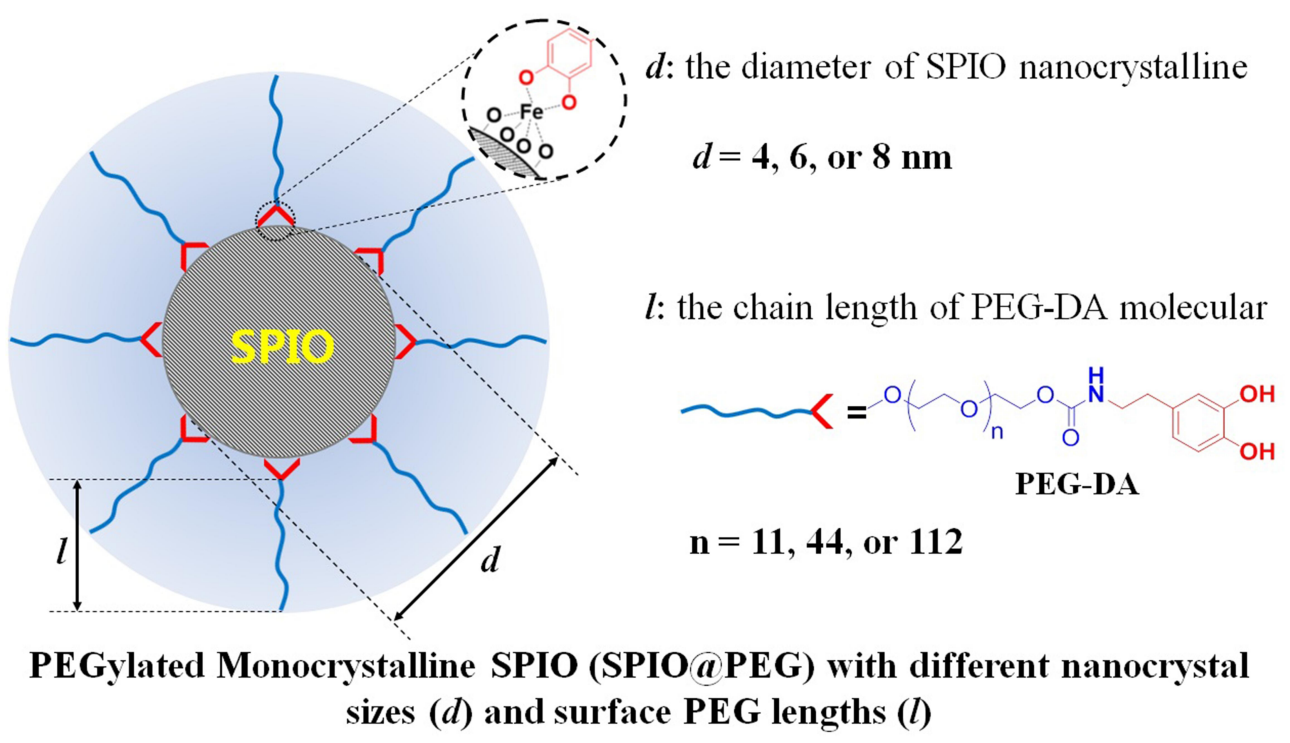

Figure I Conceptual graph of PEGylated monocrystalline SPIO nanoparticles (SPIO@PEG) with various nanocrystal sizes (d) and surface PEG lengths (I). In our research, three SPIO crystal particles $(4,6$, and $8 \mathrm{~nm})$ and three PEG $(\mathrm{Mn}=550,2 \mathrm{k}$, or 5k Da) were used to prepare nine SPIO@PEG nanoparticles.

Then, $150 \mathrm{mg}$ of mPEG-DA $(\mathrm{Mn}=550,2 \mathrm{k}$ or $5 \mathrm{k} \mathrm{Da})$ and 5 mL of SPIO@CA solution (containing 10-mg nanoparticles) were mixed and magnetically stirred under a flow of nitrogen for $72 \mathrm{~h}$ at $40^{\circ} \mathrm{C}$ after being deoxidized. The solution was dialyzed against water for 2 days to obtain PEGylated SPIO (SPIO@PEG). The concentration of iron was determined using an atomic absorption spectrometer (AAS).

\section{Relaxivity of SPIO@PEG Nanoparticles in vitro}

The samples were diluted to different iron concentrations $(0.05,0.1,0.2,0.3,0.4$, and $0.5 \mathrm{mmol} / \mathrm{L})$ and placed in test bottles. Longitudinal and transverse relaxation times $\left(T_{1}\right.$ and $T_{2}$ ) were measured at room temperature at $0.5 \mathrm{~T}$ (by a 0.5 $\mathrm{T}$ magnetic resonance developer relaxation rate analyzer, Niumag, Shanghai, China), 1.5 T and 3.0 T (by clinical GE MRI systems, General Electric Company, Boston, Massachusetts, USA), respectively. Then, $T_{1}$ or $T_{2}$ relaxivity $\left(r_{1}\right.$ or $r_{2}$ ) was calculated through the curve fitting of the relaxation rate $\left(1 / T_{1}\right.$ or $\left.1 / T_{2}, \mathrm{~s}^{-1}\right)$ versus the iron concentration $(\mathrm{mM})$.

\section{Cytotoxicity of SPIO@PEG Nanoparticles}

The mouse macrophage cell line RAW264.7 cells purchased from Procell (Wuhan, Hubei, China), were placed in 96 well plates at a concentration of $10 \times 10^{3}$ per well $(100 \mu \mathrm{L})$, and cultured for $24 \mathrm{~h}$ in a humidified atmosphere containing $5 \%$ of $\mathrm{CO}_{2}$. Then, the cells were incubated for $24 \mathrm{~h}$ in a culture medium that included SPIO@PEG nanoparticles with different Fe concentrations $(20,15,10$, and $5 \mu \mathrm{g} / \mathrm{mL})$. CCK-8 (10 $\mu \mathrm{L} /$ well) reagent (Bio Basic Inc, Konrad Cres, MO, Canada) was added under dark conditions. Absorbance was measured after $1 \sim 2 \mathrm{~h}$, and the absorbance was determined using the microplate photometer. Cell viability was calculated using the following equation: Cell viability $(\%)=\left(N_{\mathrm{s}} / N_{c}\right) \times 100$, where $N_{\mathrm{s}}$ and $N_{\mathrm{c}}$ are the absorbance of surviving cells treated with or without SPIO@PEG nanoparticles. The absorbance of media with or without SPIO@PEG nanoparticles was corrected and used as blank control for deduction.

\section{Statistical Analysis}

Data were presented as mean \pm SD (Gaussian distribution) or median and IQR (Skewed distribution). Statistical analysis was performed with SAS 9.4 (Raleigh, North Carolina, USA). Weighted ANOVA was used for comparison between groups, and the SNK-q method was used for pair-wise comparison. Two-factor ANOVA was used for factorial design. When the interaction effects were statistically significant, intra-group and inter-group pair comparisons were further conducted, and the Bonferroni method was used to adjust $\mathrm{P}$ values. The results were considered statistically significant when $\mathrm{p}<0.05$.

\section{Serum Stability Test}

SPIO@PEG (8 mmol/L Fe concentration), volumetric fraction of $20 \%$ fetal bovine serum and PBS were placed 
in $37^{\circ} \mathrm{C}$ water bath for $5 \mathrm{~min}, 1 \mathrm{~h}, 3 \mathrm{~h}, 5 \mathrm{~h}$, and $24 \mathrm{~h} .200-$ $\mu \mathrm{L}$ samples were added to $1-\mathrm{mL}$ phosphate-buffered saline (PBS) in the colorimetric dishes, and finally the particle size distribution was detected by Malvern nanoparticle size and zeta potential analyzer (Malvern, England), respectively.

\section{In vivo Imaging of Rats}

All animal procedures were performed in accordance with the Guidelines for Care and Use of Laboratory Animals of North Sichuan Medical College and experiments were approved by the Animal Ethics Committee of North Sichuan Medical College (P20191226). MRI studies were performed with a GE 3.0T MRI scanner by using a rat coil for transmission and reception of the signal. Sprague Dawley (SD) rats (age: 6-8 weeks, weight: 200 g) were anesthetized by inhalational anesthesia systems, and an MRI scan performed before and immediately after injection, 1, 30, 60, $120 \mathrm{~min}$ and $24 \mathrm{~h}$ with the contrast agent administered at a dose of $5 \mathrm{mg}(\mathrm{Fe}) / \mathrm{kg}$ body weight. Signal intensity (SI) was measured at each time point, and the change in the relative normalized signal intensities (NSI) was plotted against time. The MRI sequence and parameters were as follows.

Contrast-enhanced magnetic resonance angiography (CEMRA) imaging: $\mathrm{TE}=$ minimum, $\mathrm{TR}=6.2 \mathrm{~ms}, \mathrm{FOV}=$ $140 \mathrm{~mm} \times 140 \mathrm{~mm}$, slice thickness $=2.0 \mathrm{~mm}$, and slice spacing $=0.6 \mathrm{~mm}$, and flip angle $30^{\circ}$;

$T_{1}-T_{2}$ dual-mode imaging: $T_{1}$-mode (GRE sequence, $\mathrm{TE}=3 \mathrm{~ms}, \mathrm{TR}=9 \mathrm{~ms}$, flip angle $\left.30^{\circ}\right), T_{2}$-mode (FSE sequence, $\mathrm{TE}=76 \mathrm{~ms}, \mathrm{TR}=4.0 \mathrm{~s}$, flip angle $142^{\circ}$ ), $\mathrm{FOV}=$ $80 \mathrm{~mm} \times 80 \mathrm{~mm}$, and slice thickness $=3.0 \mathrm{~mm}$;

$T_{2}$ mapping: FSE sequence, $\mathrm{TE}=8,16,24,31,39,47$, $55,63 \mathrm{~ms}, \mathrm{TR}=1.2 \mathrm{~s}, \mathrm{FOV}=80 \mathrm{~mm} \times 80 \mathrm{~mm}$, slice thickness $=2.0 \mathrm{~mm}$, slice spacing $=0.6 \mathrm{~mm}$, and flip angle $90^{\circ}$.

After MRI scan, the rats were kept for another 4 weeks and their mental states, activities and feeds were observed. No abnormalities were found and none of them died in the end, which suggested that SPIOs have no toxicity to rats indirectly.

\section{Results}

\section{Synthesis of PEG-DA}

Catechol-group modified PEG (PEG-DA) was synthesized by a two-step chemical modification of mPEG-OH. The terminal hydroxyl group of mPEG-OH was activated by reaction with $1,1^{\prime}$-carbonyldiimidazole, and then the activated intermediate reacted with the amino group of dopamine to obtain PEG-DA (Figure S1). Three molecular weights mPEG-DA (550 Da, $2 \mathrm{k} \mathrm{Da}$ and $5 \mathrm{k} \mathrm{Da}$ ) were prepared in this work, and the corresponding ${ }^{1} \mathrm{H}$ NMR spectra can be found in the supporting information (Figures S2-S7).

\section{Synthesis of SPIO Nanocrystals with Various Crystal Sizes}

SPIO nanocrystals with high-quality were synthesized by high-temperature thermal decomposition, and the crystal size was tuned by changing the reaction solvent and heating conditions. The crystal size and dispersity were examined using transmission electron microscopy (TEM) and dynamic light scattering (DLS). As shown in Figure 2A, three SPIO nanoparticles displayed favorable monodispersity in TEM, and the lattice fringes were clearly visible in the high-resolution TEM (HRTEM) images. The crystal sizes were measured by selecting different representative areas under the TEM and were $3.7 \pm 0.7 \mathrm{~nm}$ (4-nm SPIO), $5.2 \pm 1.2 \mathrm{~nm}(6-\mathrm{nm}$ SPIO), and $6.7 \pm 0.9 \mathrm{~nm}$ (8-nm SPIO), respectively. In the DLS analysis, the SPIO nanoparticles show narrow particle size distribution in hexane (PDI: $0.421,0.356$ and 0.283 for $4 \mathrm{~nm}, 6 \mathrm{~nm}$ and $8 \mathrm{~nm}$ SPIO nanoparticles, respectively), and there is a slight increase in the average size $(4.4 \pm 3.0 \mathrm{~nm}, 11.2 \pm 6.0 \mathrm{~nm}$ and $16.0 \pm$ $10.0 \mathrm{~nm}$, respectively) due to the surface organic molecular layer. Selected area electron diffraction (SAED) patterns of this SPIO nanocrystals are shown in Figure S8a$\underline{\mathrm{S} 8 \mathrm{c}}$. The measured lattice spacing based on the rings in the diffraction pattern conformed to the known lattice spacing for $\mathrm{Fe}_{3} \mathrm{O}_{4}$ crystal (Figure $\mathrm{S} 8 \mathrm{~d}$ ).

\section{Synthesis of PEGylated SPIO with Different Crystal Sizes and PEG Lengths}

SPIO nanoparticles were transferred to the aqueous phase by ligand exchange with sodium citrate. Then, the PEGDA with different molecular weights $(550,2 \mathrm{k}$ and $5 \mathrm{k} \mathrm{Da})$ were chelated onto the surface of the SPIO nanoparticles in water. The schematic preparation of PEGylated SPIO nanoparticles is shown in Figure S9. Nine PEGylated SPIO samples with different crystal sizes and PEG lengths were prepared. The particle size distribution of the PEGylated SPIO samples in aqueous solution was measured by DLS and is shown in Figure 2C. The average particle size was calculated and is shown in Table S1. 

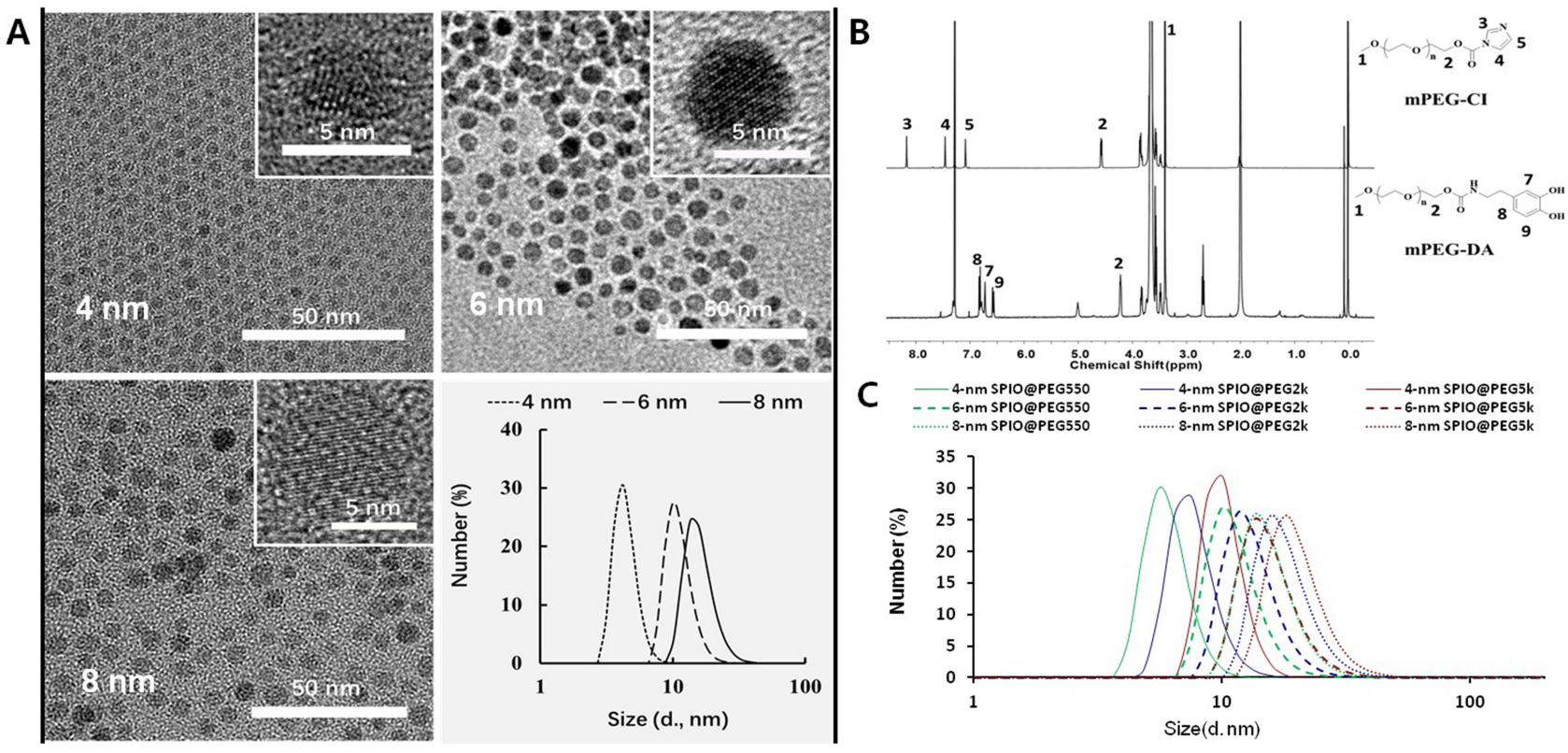

Figure 2 Size and PEG length-controlled PEGylated monocrystalline SPIO (SPIO@PEG) Nanocomposite. (A) TEM, HRTEM and DLS distribution of three SPIO nanocrystals (in hexane). (B) Chemical characterization of polyethylene glycol dopamine-modification. ' $\mathrm{H}$ NMR spectra $\left(\mathrm{CDCl}_{3}\right)$ and characteristic peak assignment of activated intermediate (PEG-Cl) and end product (PEG-DA). (C) DLS diameter distribution of the nine PEGylated SPIO nanoparticles in water.

\section{Relaxivities of PEGylated SPIO at Different Magnetic Fields}

Relaxivities $\left(r_{1}\right.$ and $r_{2}$ ) of PEGylated SPIO were measured at three main magnetic fields $(0.5 \mathrm{~T}, 1.5 \mathrm{~T}$, and $3.0 \mathrm{~T})$, and are listed in Table 1. $r_{1}$ and $r_{2}$ increased with an increase in the size of the SPIO nanocrystal, and this change is more sensitive for $r_{2}$ which results in an increase of the $r_{2} / r_{1}$ ratio. With the increasing molecular weight of PEG, $r_{1}$ and $r_{2}$ of the PEGylated SPIO nanoparticles also increase. Moreover, regular changes were observed at the three different magnetic fields. For all PEGylated SPIO, $r_{1}$ significantly reduced with the increase in the magnetic intensity, whereas $r_{2}$ slightly increased, leading to a sharp increase in the $r_{2} / r_{1}$ ratio.

\section{Serum Stability and Cytotoxicity of PEGylated SPIO in vitro}

We monitored the changes in the size of the nine PEGylated SPIO in DLS after incubation with $20 \%(\mathrm{v} / \mathrm{v})$ fetal bovine serum (FBS) solution at $37^{\circ} \mathrm{C}$ for $24 \mathrm{~h}$. All the data were statistically analyzed, and there were statistical differences among some of the data, but we further compared the mean (Number) and median (Intensity) of particle size at different time points and found no significant changes in the mean or median at each time point (4-nm SPIO@PEG550 as an example in Table S2A). However, particle sizes over $100 \mathrm{~nm}$ gradually appeared over time (Figures S10-S12), though visible precipitation did not occur throughout the experiment. The cytotoxicity of PEGylated SPIO nanoparticles was evaluated by CCK-8 assay on the mouse macrophage cell line Raw 264.7. After being incubated with the nine PEGylated SPIO nanoparticles for $24 \mathrm{~h}$, the cells were collected for visual inspection under a microscope. No observable morphological abnormalities were noted. Figures S13-S15 show the cytotoxicity of the nine PEGylated SPIO nanoparticles after incubation for $24 \mathrm{~h}$. After statistical analysis, there was no statistically significant difference in cytotoxicity between different concentrations in nine samples (4-nm SPIO@PEG550 as an example in Table S2B, P = 0.256). The results suggest that the PEGylated SPIO nanoparticles possess reliable biocompatibility in our measured range $(20-\mu \mathrm{g} \mathrm{Fe} / \mathrm{mL})$.

\section{In vivo MRI Studies of PEGylated SPIO CEMRA of SD Rats}

CEMRA of SD rats was carried out on a clinical 3.0T MRI scanner, and the clinical used contrast agent (Magnevist) is used as control. Figure 3 and Figure S16 show the MR images of SD rats before and after intravenous injection of 4-nm SPIO@PEG5k, 6-nm SPIO@PEG5k, 8-nm SPIO@PEG5k nanoparticles, or Magnevist with the CEMRA sequence. The enhancement of angiography is seen for all but displays 
Table I Relaxivities of Nine PEGylated SPIO Nanoparticles and Commercial Contrast Agent (Magnevist and Ferumoxytol) at Different Main Magnetic Fields

\begin{tabular}{|c|c|c|c|c|c|c|c|c|c|}
\hline \multirow[t]{2}{*}{ Sample } & \multicolumn{3}{|l|}{$0.5 \mathrm{~T}$} & \multicolumn{3}{|l|}{$1.5 \mathrm{~T}$} & \multicolumn{3}{|l|}{$3.0 \mathrm{~T}$} \\
\hline & $r_{1}\left(\mathrm{mM}^{-1} \mathrm{~s}^{-1}\right)$ & $r_{2}\left(m M^{-1} s^{-1}\right)$ & $r_{2} / r_{1}$ & $r_{1}\left(m^{-1} s^{-1}\right)$ & $r_{2}\left(m M^{-1} s^{-1}\right)$ & $r_{2} / r_{1}$ & $r_{1}\left(\mathrm{mM}^{-1} \mathrm{~s}^{-1}\right)$ & $r_{2}\left(m M^{-1} s^{-1}\right)$ & $r_{2} / r_{1}$ \\
\hline 4-nm SPIO@PEG550a & 14.2 & 24.6 & 1.73 & 11.5 & 35.5 & 3.08 & 6.99 & 41.5 & 5.94 \\
\hline $\begin{array}{l}\text { 4-nm } \\
\text { SPIO@PEG2k }\end{array}$ & 13.5 & 22.5 & 1.67 & 11.4 & 30.9 & 2.71 & 7.57 & 33.1 & 4.38 \\
\hline $\begin{array}{l}\text { 4-nm } \\
\text { SPIO@PEG5k }\end{array}$ & 16.1 & 28.0 & 1.74 & 14.2 & 40.5 & 2.85 & 8.82 & 46.2 & 5.24 \\
\hline $\begin{array}{l}\text { 6-nm } \\
\text { SPIO@PEG550 }\end{array}$ & 28.1 & 54.9 & 1.95 & 14.2 & 67.5 & 4.76 & 6.84 & 67.9 & 9.92 \\
\hline $\begin{array}{l}\text { 6-nm } \\
\text { SPIO@PEG2k }\end{array}$ & 29.1 & 63.1 & 2.17 & 16.9 & 70.6 & 4.17 & 8.36 & 72.2 & 8.63 \\
\hline $\begin{array}{l}\text { 6-nm } \\
\text { SPIO@PEG5k }\end{array}$ & 34.2 & 70.1 & 2.05 & 19.8 & 77.8 & 3.93 & 10.2 & 81.4 & 7.96 \\
\hline $\begin{array}{l}\text { 8-nm } \\
\text { SPIO@PEG550 }\end{array}$ & 41.6 & 102.7 & 2.47 & 17.2 & 117.1 & 6.81 & 7.94 & 119.4 & 15.1 \\
\hline $\begin{array}{l}\text { 8-nm } \\
\text { SPIO@PEG2k }\end{array}$ & 45.3 & 111.0 & 2.45 & 19.0 & 122.0 & 6.42 & 8.60 & 129.5 & 15.1 \\
\hline $\begin{array}{l}\text { 8-nm } \\
\text { SPIO@PEG5k }\end{array}$ & 47.3 & 118.1 & 2.49 & 20.0 & 130.1 & 6.52 & 10.1 & 141.4 & 14.0 \\
\hline $\begin{array}{l}\text { Magnevist } \\
\text { (GdDTPA) }\end{array}$ & 3.72 & 4.29 & 1.15 & 3.56 & 4.08 & 1.15 & 3.58 & 3.96 & 1.11 \\
\hline Ferumoxytol $^{b}$ & 38.0 & 85 & 2.24 & 19.9 & 60.8 & 3.06 & 10.0 & 62.3 & 6.23 \\
\hline
\end{tabular}

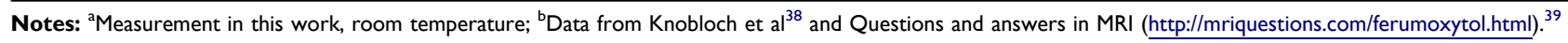

different effects. High contrast imaging for an extended period (more than 2 h) is obtained in the 4-nm SPIO@PEG5k group, whereas the enhancement is relatively poor and quickly disappears in the other groups. The enhancement disappeared within 30 min in the 8-nm SPIO@PEG5k group, and disappeared within a few minutes in Magnevist group.

\section{$T_{1}-T_{2}$ Dual-Mode MRI in Liver}

Previous studies of relaxivity in vitro have found that 4-nm SPIO@PEG nanoparticles are a typical $T_{1}-T_{2}$ dual-mode contrast agent at $3.0 \mathrm{~T}\left(r_{2} / r_{1}=5.24\right)$. To verify its validity in vivo, $T_{1}-T_{2}$ dual-mode MRI of SD rats was performed with a clinical 3.0 T MRI scanner. The SD rats liver underwent $T_{1}$ WI and $T_{2} \mathrm{WI}$ in tandem before and after $(1,30$, and $120 \mathrm{~min})$ intravenous injection of 4-nm SPIO@PEG5k nanoparticles, with 8-nm SPIO@PEG5k nanoparticles as control. Figure 4 shows the images and corresponding NSI. The results show that the liver tissue reveals a hyperintensity in $T_{1} \mathrm{WI}$ and a hypointensity in $T_{2} \mathrm{WI}$ after administration of the 4-nm SPIO@PEG5k, and the hepatic vessels are visible. The contrast enhancement effect lasts for more than $2 \mathrm{~h}$ in the $T_{1}-T_{2}$ dual-mode imaging. On administration of the 8-nm SPIO@PEG5k nanoparticles, there is only a slight enhancement in $T_{1} \mathrm{WI}$ at $1 \mathrm{~min}$ and a strong reduction in $T_{2} \mathrm{WI}$.

\section{MRI $T_{2}$ Mapping in Liver}

Transverse $T_{2}$ mapping in liver of SD rats proceeded before and after administration of SPIO@PEG nanoparticles (Figure 5A). Figure 5B depicts the mean $T_{2}$ values of liver parenchyma at different time points and shows obvious changes, and $T_{2}$ maintained lower values for a prolonged time in both 4-nm SPIO@PEG5k and 8-nm SPIO@PEG5k groups.

\section{Discussion}

PEGylated SPIOs were developed over the past decades and may be the most promising alternative to GBCAs in MRI contrast agent. However, there was a lack of systematic study on the effects of particle size, PEG length, and magnetic field strength on its imaging. In our study, we 

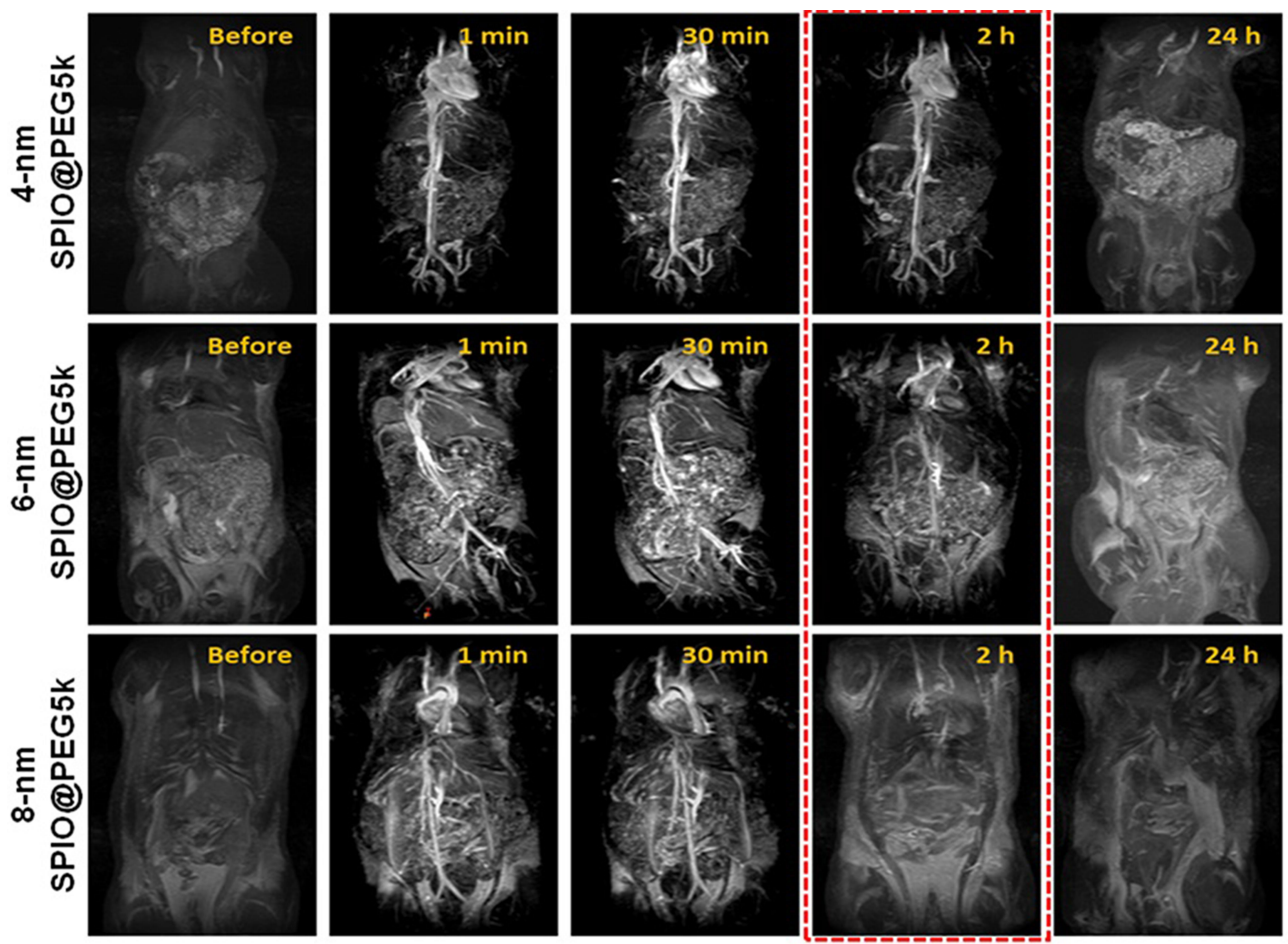

Figure 3 MRA images of SD rats before and after intravenous injection of SPIO@PEG nanoparticles. High contrast imaging more than $2 \mathrm{~h}$ is obtained in the 4-nm SPIO@PEG5k group.
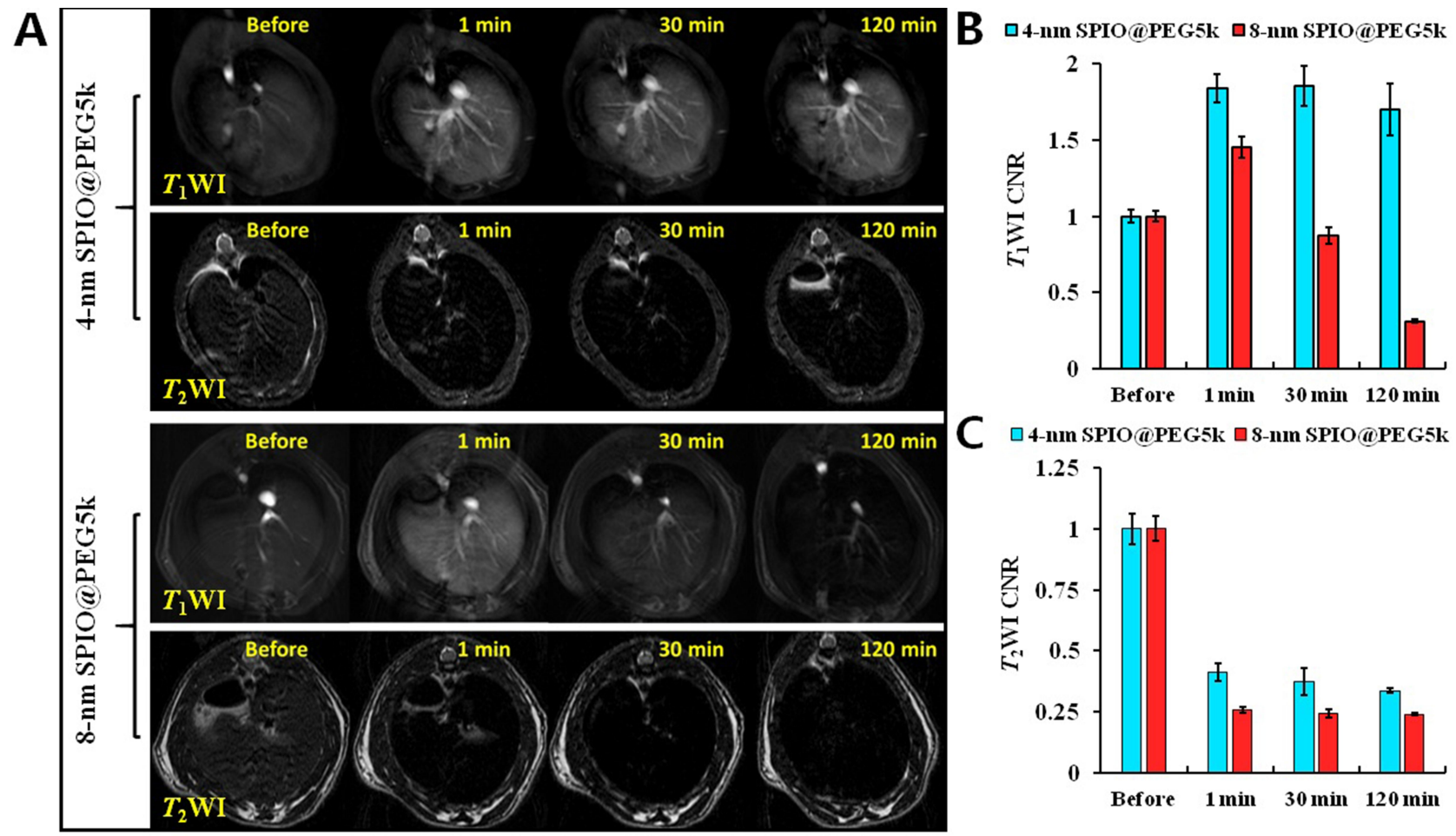

Figure 4 (A) SD rats hepatic $T_{1}-T_{2}$ dual-mode imaging before and after intravenous injection of 4-nm SPIO@PEG5k or 8-nm SPIO@PEG5k; (B) NSI in liver parenchyma on $T_{1} \mathrm{Wl}$; (C) NSI in liver parenchyma on $T_{2} \mathrm{Wl}$. 


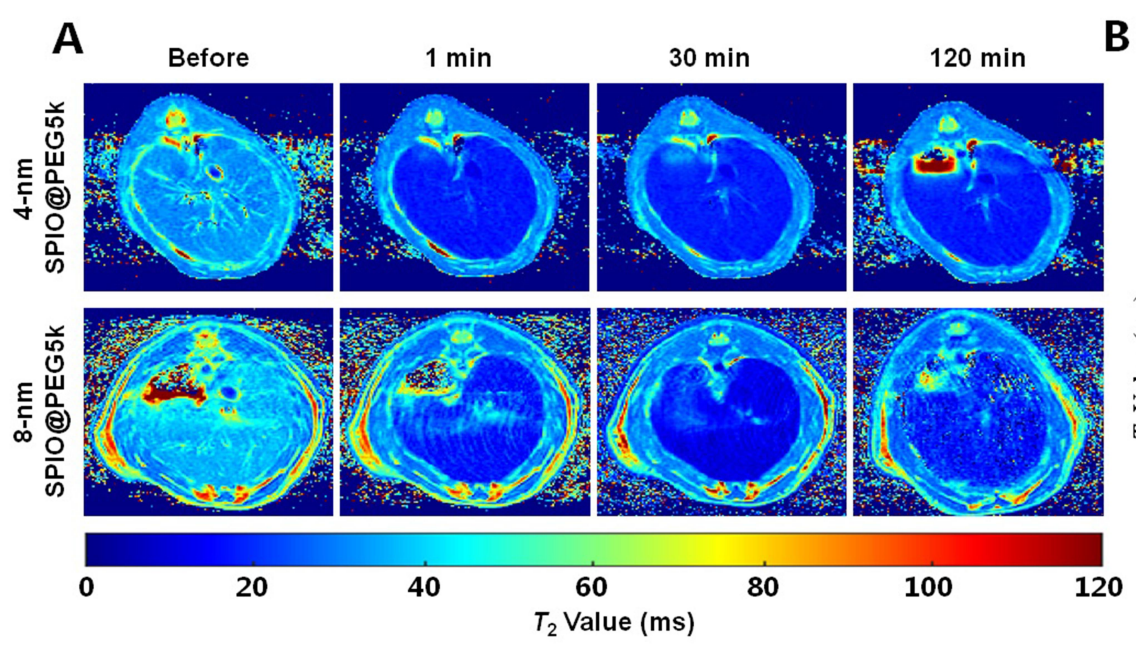

B
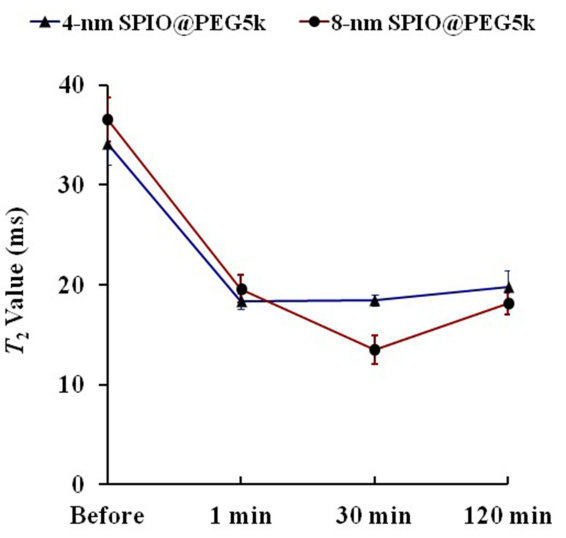

Figure 5 (A) Transverse $T_{2}$ mapping of SD rat liver, (B) Mean $T_{2}$ values in liver parenchyma before and after intravenous injection of 4-nm SPIO@PEG5k or 8-nm SPIO@PEG5k.

successfully prepared nine PEGylated monocrystalline SPIO with different crystal sizes and PEG lengths firstly, and then designed systematic experiments to study their performances and characteristics as MRI contrast agent.

To obtain PEGylated monodispersion SPIO, catecholgroup modified PEG (mPEG-DA) was designed and coated on the surface of inorganic SPIO nanocrystals by the coordination of catechol and $\mathrm{Fe}^{3+}$ (Figure 1), which was commonly used in previous studies. ${ }^{28,29}$ MPEG-DA was synthesized by a two-step chemical modification of mPEG-OH, and confirmed by ${ }^{1} \mathrm{H}$ NMR spectra of the activated intermediate and the final product. The characteristic peaks of PEG (1 and 2) and the imidazole ring (3, 4 , and 5) appear together in the ${ }^{1} \mathrm{H}$ NMR spectrum of mPEG-CI showing the activation of the terminal hydroxyl group, and disappearance of the imidazole ring peaks and appearance of the dopamine peaks $(7,8$, and 9) in the ${ }^{1} \mathrm{H}$ NMR spectrum of mPEG-DA (Figure 2B). Then, mPEG-DA with different molecular weights $(550,2 \mathrm{k}$ and $5 \mathrm{k} \mathrm{Da})$ were chelated onto the surface of three SPIO nanocrystals with different size, respectively, to obtain nine PEGylated SPIO samples with different crystal sizes and PEG lengths. These SPIO nanoparticles show narrow particle size distribution in the aqueous phase and the size increases with crystal size and the molecular weight of PEG-DA (Figure 2C). It demonstrated that the longer PEG molecules can create a thicker hydration layer on the surface of the SPIO nanocrystals.

$T_{1}$ relaxivity $\left(r_{1}\right)$ and $T_{2}$ relaxivity $\left(r_{2}\right)$ are the reciprocal of $T_{1}$ and $T_{2}$ relaxation time per unit concentration of contrast agents, and represent the net effectiveness of shorter $T_{1}$ and $T_{2}$ relaxation times in MRI. $r_{2} / r_{1}$ ratio can be used to classify a contrast agent as a $T_{1}, T_{2}$, or $T_{1}-T_{2}$ dual-mode contrast agent in MRI enhancement application. Generally, $T_{1}$ contrast agents have a low $r_{2} / r_{1}$ ratio and can produce bright images in a $T_{1}$-weighted sequence, like paramagnetic chelates of gadolinium $\left(\mathrm{Gd}^{3+}\right)$, iron $\left(\mathrm{Fe}^{3}\right.$ $\left.{ }^{+}\right)$, or manganese $\left(\mathrm{Mn}^{2+}\right)$ ions $\left(r_{2} / r_{1}\right.$ ratio approximately equal to 1$){ }^{3} T_{2}$ contrast agents possess a high $r_{2} / r_{1}$ ratio (greater than 10) and can produce dark images in a $T_{2}$ or $T_{2}{ }^{*}$-weighted sequence, like most of the magnetic nanoparticles. ${ }^{13}$ The ideal $T_{1}-T_{2}$ dual-mode contrast agent should have an intermediate $r_{2} / r_{1}$ ratio $(5 \sim 8)$, and hence can be used for contrast enhancement in $T_{1}-T_{2}$ dual-mode MRI. ${ }^{30}$ Ferumoxytol is one of the SPIO commercial products with an average of $30 \mathrm{~nm}$ size. Its $r_{1}, r_{2}$ and $r_{2} / r_{1}$ are similar to the 6-nm SPIO sample synthesized in our study (Table 1), but the crystal shape and stability are not so excellent, on account of synthesized by coprecipitation method. Ferumoxytol is more likely to be captured by the reticuloendothelial system and has a shorter half-life of blood. Relaxivities of PEGylated SPIO at different magnetic fields show that the crystal size and the PEG length significantly influence the relaxivities of the PEGylated SPIO nanoparticles. The 8-nm SPIO@PEG5k has extremely high $r_{1}$ and $r_{2}$, is a good $T_{2}$ contrast agent at $3.0 \mathrm{~T}\left(r_{2} / r_{1}=14.0\right)$, is an ideal $T_{1}-T_{2}$ dual-mode contrast agent at $1.5 \mathrm{~T}\left(r_{2} / r_{1}=6.52\right)$, and is also an effective $T_{1}$ contrast agent at $0.5 \mathrm{~T}\left(r_{2} / r_{1}=2.49\right)$; 4-nm SPIO@PEG5k also has high $r_{1}$ and $r_{2}$, is $T_{1}-T_{2}$ dual-mode contrast agent at $3.0 \mathrm{~T}\left(r_{2} / r_{1}=5.24\right)$, and is a good $T_{1}$ contrast agent at $0.5 \mathrm{~T}\left(r_{2} / r_{1}=1.74\right)$ and $1.5 \mathrm{~T}\left(r_{2} / r_{1}=2.85\right)$. These results 
indicate that it depends not only on the material itself but also on the main magnetic field strength for a contrast agent to be effective as $T_{1}, T_{2}$, or $T_{1}-T_{2}$ dual-mode contrast agent in MRI. However, this is often overlooked in previous reports. Consequently, the PEGylated SPIOs display good relaxivity but demonstrate different enhancement features. PEGylated SPIOs with different nanocrystal sizes and surface PEG lengths can be used as $T_{1}, T_{2}$, or $T_{1}-T_{2}$ dual-mode contrast agent at the appropriate magnetic field in MRI enhancement application.

The biodistribution and clearance of nanoparticles were heavily dependent on their stability and their interactions with proteins in plasma upon entering the bloodstream. Regarding serum stability test results of SPIO, statistical differences were found between some different sizes at different time. However, the differences had no clinical significance, because of the tiny change of mean particle sizes, which may be caused by attaching of major components of serum to SPIO, like proteins. PEGylated SPIO creates a PEG hydration layer on the surface of the SPIO nanocrystals, which can prevent non-specific binding to proteins in plasma. This is also an important factor impacting the imaging effect and characteristics for MRI nano contrast agents. In addition, all the nine PEGylated SPIO samples showed no statistical toxic effect on mouse macrophage cell line Raw 264.7, which suggested that PEGylated SPIO samples have good biological safety.

To evaluate the contrast enhancement of different PEGylated SPIO in vivo, three typical MR imaging sequences were used to study SD rats at a GE 3.0 T MRI scanner. CEMRA is an important clinical tool to detect cardiovascular diseases, such as vascular malformation, myocardial infarction, atherosclerotic plaque, and tumor angiogenesis. ${ }^{31-34}$ Usually, the CEMRA sequence uses ultrashort TE and TR to enable a high $T_{1}$ weighting and to obtain high-resolution blood vessel imaging after administration of an intravenous $T_{1}$ contrast agent. The CEMRA of SD rats is consistent with the in vitro data, 4-nm SPIO@PEG5k is a $T_{1}$ - $T_{2}$ dual-mode contrast agent, while 8-nm SPIO@PEG5k is only a $T_{2}$ contrast agent at $3.0 \mathrm{~T}$. Another reason is that larger particles are more easily cleared by the reticuloendothelial system, which leads to a short circulation time for 8-nm SPIO@PEG5k. Therefore, 4-nm SPIO@PEG5k is more suitable for CEMRA in MRI. $T_{1}-T_{2}$ dual-mode MRI can easily produce an accurate match of spatial and temporal imaging parameters and provide cross-validation information. ${ }^{35,36}$ As a result, it is routinely performed in clinical diagnostics. The results of $T_{1}-T_{2}$ dual-mode MRI in rats' liver confirm that the 4-nm SPIO@PEG5k can be used as a $T_{1}-T_{2}$ dual-mode contrast agent. However, the 8-nm SPIO@PEG5k is only a $T_{2}$ contrast agent at $3.0 \mathrm{~T}$ MRI. The $T_{2}$ value is a characteristic parameter of biological tissues, and accurate determination of the $T_{2}$ value is helpful to enhance disease detection and monitoring. It is used in dynamic contrast agent studies, diagnosis of epilepsy, and determination of the severity of Parkinson's disease in clinical practice. ${ }^{37}$ These data of MRI $T_{2}$ mapping in rats' liver illustrate that 4-nm SPIO@PEG5k and 8-nm SPIO@PEG5k have specific liver distribution for a long period.

We successfully prepared nine PEGylated monocrystalline SPIO nanoparticles with different nanocrystal sizes and surface PEG lengths. All these PEGylated SPIO samples exhibit high relaxivities $\left(r_{1}\right.$ and $\left.r_{2}\right)$ and have different enhancement features which are related to their crystal size and PEG length. The 8-nm SPIO@PEG5k is an effective $T_{2}$ contrast agent at $3.0 \mathrm{~T}\left(r_{2} / r_{1}=14.0\right)$, is an ideal $T_{1}-T_{2}$ dual-mode contrast agent at $1.5 \mathrm{~T}\left(r_{2} / r_{1}=6.52\right)$, and is also an effective $T_{1}$ contrast agent at $0.5 \mathrm{~T}\left(r_{2} / r_{1}=2.49\right)$. 4-nm SPIO@PEG5k is a $T_{1}-T_{2}$ dual-mode contrast agent at $3.0 \mathrm{~T}\left(r_{2} / r_{1}=5.24\right)$, and is a good $T_{1}$ contrast agent at $0.5 \mathrm{~T}\left(r_{2} / r_{1}=1.74\right)$ or $1.5 \mathrm{~T}\left(r_{2} / r_{1}=2.85\right)$. In MRI studies of SD rats at 3.0 T, 4-nm SPIO@PEG5k shows excellent contrast enhancement in MRA, $T_{1}-T_{2}$ dual-mode imaging, and $T_{2}$ mapping, whereas 8-nm SPIO@PEG5k only displays good enhancement in $T_{2} \mathrm{WI}$ and $T_{2}$ mapping.

\section{Conclusions}

In summary, PEGylated SPIOs synthesized in this study showed high $T_{1}$ and $T_{2}$ relaxivity, good biosafety and stability, and are the promising contrast agents in MRI. Furthermore, PEGylated SPIOs with different nanocrystal sizes and surface PEG lengths showed different imaging characteristics at various magnetic field intensity and can be used as $T_{1}, T_{2}$, or $T_{1}-T_{2}$ dual-mode contrast agents to meet the clinical demands of MRI at specific magnetic fields. Our results can provide a foundation for researchers in the selection of nanocrystal size, PEG length and magnetic field intensity for further study.

\section{Abbreviations}

GBCAs, gadolinium-based contrast agents; MRI, magnetic resonance imaging; NSF, nephrogenic system fibrosis; SPIO, Superparamagnetic iron oxides; PEG, polyethylene glycol; SNR, signal-to-noise ratio; CEMRI, contrastenhanced MRI; AAS, atomic absorption spectrometer; PBS, phosphate-buffered saline; SD, Sprague Dawley; 
SI, Signal intensity; NSI, normalized signal intensities; TEM, transmission electron microscopy; DLS, dynamic light scattering; HRTEM, high-resolution TEM; SAED, Selected area electron diffraction; FBS, fetal bovine serum.

\section{Acknowledgment}

The work was supported by National Natural Science Foundation of China (81601490), Science and Technology Project of Sichuan, China (2016JY0172), Science and Technology Project of Municipal School Strategic Cooperation, Nanchong (NSMC20170430), Scientific Research Start-up Fund of North Sichuan Medical College (CBY15-QD02), Pre-research Project of North Sichuan Medical College (CBY19-YZ05). We would like to thank Editage (www.editage.cn) for English language editing.

\section{Disclosure}

The authors report there are no conflicts of interest to declare.

\section{References}

1. Fingerhut S, Sperling M, Holling M, et al. Gadolinium-based contrast agents induce gadolinium deposits in cerebral vessel walls, while the neuropil is not affected: an autopsy study. Acta Neuropathol. 2018;136(1):127-138. doi:10.1007/s00401-018-1857-4

2. Kanda T, Fukusato T, Matsuda M, et al. Gadolinium-based contrast agent accumulates in the brain even in subjects without severe renal dysfunction: evaluation of autopsy brain specimens with inductively coupled plasma mass spectroscopy. Radiology. 2015;276(1):228-232. doi:10.1148/radiol.2015142690

3. Runge VM, Heverhagen JT. Advocating the development of next-generation high-relaxivity gadolinium chelates for clinical magnetic resonance. Invest Radiol. 2018;53:381-389.

4. Laurent S, Forge D, Port M, et al. Magnetic iron oxide nanoparticles: synthesis, stabilization, vectorization, physicochemical characterizations, and biological applications. Chem Rev. 2008;108 (6):2064-2110. doi:10.1021/cr068445e

5. Lee SH, Kim BH, Na HB, Hyeon T. Paramagnetic inorganic nanoparticles as T1 MRI contrast agents. Wiley Interdiscip Rev Nanomed Nanobiotechnol. 2014;6(2):196-209. doi:10.1002/wnan.1243

6. Wang Y-XJ. Superparamagnetic iron oxide based MRI contrast agents: current status of clinical application. Quant Imaging Med Surg. 2011;1(1):35-40.

7. Xie J, Liu G, Eden HS, Ai H, Chen X. Surface-engineered magnetic nanoparticle platforms for cancer imaging and therapy. Acc Chem Res. 2011;44(10):883-892. doi:10.1021/ar200044b

8. Su H-Y, Wu C-Q, Li D-Y, Ai H. Self-assembled superparamagnetic nanoparticles as MRI contrast agents- a review. Chin Phys $B$. 2015;24:12. doi:10.1088/1674-1056/24/12/127506

9. Fu J, Fiegel J, Krauland E, Hanes J. New polymeric carriers for controlled drug delivery following inhalation or injection. Biomaterials. 2002;23(22):4425-4433. doi:10.1016/S0142-9612(02)00182-5

10. Cheng T-C, Chuang K-H, Chen M, et al. Sensitivity of PEGylated interferon detection by anti-polyethylene glycol (PEG) antibodies depends on PEG length. Bioconjug Chem. 2013;24(8):1408-1413. doi: $10.1021 / \mathrm{bc} 3006144$
11. Knop K, Hoogenboom R, Fischer D, Schubert US. Poly(ethylene glycol) in drug delivery: pros and cons as well as potential alternatives. Angew Chem Int Ed. 2010;49(36):6288-6308. doi:10.1002/anie.200902672

12. Seifert O, Pollak N, Nusser A, et al. Immuno-LipoTRAIL: targeted delivery of TRAIL-functionalized liposomal nanoparticles. Bioconjug Chem. 2014;25(5):879-887. doi:10.1021/bc400517j

13. Ai H, Flask C, Weinberg B, et al. Magnetite-loaded polymeric micelles as ultrasensitive magnetic-resonance probes. Adv Mater. 2005;17(16):1949-1952. doi:10.1002/adma.200401904

14. Li L, Wu C, Pan L, et al. Bombesin-functionalized superparamagnetic iron oxide nanoparticles for dual-modality MR/NIRFI in mouse models of breast cancer. Int $J$ Nanomedicine. 2019;14:6721-6732. doi:10.2147/IJN.S211476

15. Nasongkla N, Shuai X, Ai H, et al. cRGD-functionalized polymer micelles for targeted doxorubicin delivery. Angew Chem Int Ed Engl. 2004;43(46):6323-6327. doi:10.1002/anie.200460800

16. Amstad E, Gillich T, Bilecka I, Textor M, Reimhult E. Ultrastable iron oxide nanoparticle colloidal suspensions using dispersants with catechol-derived anchor groups. Nano Lett. 2009;9(12):4042-4048. doi: $10.1021 / \mathrm{nl} 902212 \mathrm{q}$

17. Kim BH, Lee N, Kim H, et al. Large-scale synthesis of uniform and extremely small-sized iron oxide nanoparticles for high-resolution T1 magnetic resonance imaging contrast agents. $J$ Am Chem Soc. 2011;133(32):12624-12631. doi:10.1021/ja203340u

18. Na HB, Palui G, Rosenberg JT, Ji X, Grant SC, Mattoussi H. Multidentate catechol-based polyethylene glycol oligomers provide enhanced stability and biocompatibility to iron oxide nanoparticles. ACS Nano. 2012;6(1):389-399. doi:10.1021/nn203735b

19. Sandiford L, Phinikaridou A, Protti A, et al. Bisphosphonate-anchored PEGylation and radiolabeling of superparamagnetic iron oxide: long-circulating nanoparticles for in vivo multimodal (T1 MRI-SPECT) imaging. ACS Nano. 2013;7(1):500-512. doi:10.1021/nn3046055

20. Bulte JWM, Kraitchman DL. Iron oxide MR contrast agents for molecular and cellular imaging. NMR Biomed. 2004;17(7):484-499. doi:10.1002/nbm.924

21. Lee N, Yoo D, Ling D, Cho MH, Hyeon T, Cheon J. Iron oxide based nanoparticles for multimodal imaging and magnetoresponsive therapy. Chem Rev. 2015;115(19):10637-10689. doi:10.1021/acs. chemrev.5b00112

22. Bai C, Jia Z, Song L, et al. Time-dependent T1-T2 switchable magnetic resonance imaging realized by $\mathrm{c}(\mathrm{RGDyK})$ modified ultrasmall Fe3O4 nanoprobes. Adv Funct Mater. 2018;28(32):1802281. doi:10.1002/adfm.201802281

23. Zhou Z, Bai R, Munasinghe J, Shen Z, Nie L, Chen X. T1-T2 dual-modal magnetic resonance imaging: from molecular basis to contrast agents. ACS Nano. 2017;11(6):5227-5232. doi:10.1021/acsnano.7b03075

24. Cai Z, Wu C, Yang L, Wang D, Ai H. Assembly-controlled magnetic nanoparticle clusters as MRI contrast agents. ACS Biomater Sci Eng. 2020. doi:10.1021/acsbiomaterials.9b01198

25. Demortière A, Panissod P, Pichon BP, et al. Size-dependent properties of magnetic iron oxide nanocrystals. Nanoscale. 2011;3 (1):225-232. doi:10.1039/C0NR00521E

26. Tong S, Hou S, Zheng Z, Zhou J, Bao G. Coating optimization of superparamagnetic iron oxide nanoparticles for high T2 relaxivity. Nano Lett. 2010;10(11):4607-4613. doi:10.1021/n1102623x

27. Sun S, Zeng H, Robinson DB, et al. Monodisperse MFe2O4 ( $\mathrm{M}=\mathrm{Fe}$, Co, Mn) nanoparticles. J Am Chem Soc. 2004;126(1):273-279. doi: $10.1021 / \mathrm{ja} 0380852$

28. Li Y, Wen J, Qin M, Cao Y, Ma H, Wang W. Single-molecule mechanics of catechol-iron coordination bonds. ACS Biomater Sci Eng. 2017;3(6):979-989. doi:10.1021/acsbiomaterials.7b00186

29. Xu C, Xu K, Gu H, et al. Dopamine as a robust anchor to immobilize functional molecules on the iron oxide shell of magnetic nanoparticles. $J$ Am Chem Soc. 2004;126(32):9938-9939. doi: $10.1021 / \mathrm{ja} 0464802$ 
30. Zhou Z, Zhao Z, Zhang $\mathrm{H}$, et al. Interplay between longitudinal and transverse contrasts in $\mathrm{Fe} 3 \mathrm{O} 4$ nanoplates with (111) exposed surfaces. ACS Nano. 2014;8(8):7976-7985. doi:10.1021/nn5038652

31. Jain R, Sawhney S. Contrast-enhanced MR angiography (CE-MRA) in the evaluation of vascular complications of renal transplantation. Clin Radiol. 2005;60(11):1171. doi:10.1016/j.crad.2005.05.004

32. Svensson J. Contrast-enhanced magnetic resonance angiography. Acta Radiol. 2015;44(s429):1-30. doi:10.1034/j.1600-0455.44. s.429.1.x

33. Wu C, Li D, Yang L, et al., Multivalent manganese complex decorated amphiphilic dextran micelles as sensitive MRI probes. J Mater Chem B. 2015;8:1470-1473. doi:10.1039/C4TB02036G

34. Wu C, Yang L, Chen Z, et al. Poly(ethylene glycol) modified Mn2+ complexes as contrast agents with a prolonged observation window in rat MRA. RSC $A d v$. 2017;7(86):54603-54609. doi:10.1039/ c7ra09975d
35. Shin T-H, Choi J-S, Yun S, et al. T1 and T2 dual-mode MRI contrast agent for enhancing accuracy by engineered nanomaterials. ACS Nano. 2014;8(4):3393-3401. doi:10.1021/nn405977t

36. Zhou Z, Wu C, Liu H, et al. Surface and interfacial engineering of iron oxide nanoplates for highly efficient magnetic resonance angiography. ACS Nano. 2015;9(3):3012-3022. doi:10.1021/ nn507193f

37. Deoni SCL, Rutt BK, Peters TM. Rapid combined T1 and T2 mapping using gradient recalled acquisition in the steady state. Magn Reson Med. 2003;49(3):515-526. doi:10.1002/mrm.10407

38. Knobloch G, Colgan T, Wiens CN, et al. Relaxivity of Ferumoxytol at $1.5 \mathrm{~T}$ and $3.0 \mathrm{~T}$. Invest Radiol. 2018;53(5):257-263. doi:10.1097/ RLI.0000000000000434

39. Questions and answers in MRI. Available from: http://mriquestions. com/ferumoxytol.html.

\section{Publish your work in this journal}

The International Journal of Nanomedicine is an international, peerreviewed journal focusing on the application of nanotechnology in diagnostics, therapeutics, and drug delivery systems throughout the biomedical field. This journal is indexed on PubMed Central, MedLine, CAS, SciSearch ${ }^{\mathbb{2}}$, Current Contents ${ }^{\mathbb{R}} /$ Clinical Medicine,
Journal Citation Reports/Science Edition, EMBase, Scopus and the Elsevier Bibliographic databases. The manuscript management system is completely online and includes a very quick and fair peer-review system, which is all easy to use. Visit http://www.dovepress.com/ testimonials.php to read real quotes from published authors. 